\title{
Rotary Encoder Magnet Inspection with Noise Elimination
}

\author{
Dr. Koen Vervaeke \\ Magcam NV, Romeinse straat 18, B-3001 Leuven, Belgium \\ koen.vervaeke@magcam.com
}

\begin{abstract}
Summary:
We report on a method to measure a noise-free magnetic field distribution of rotary encoder sensor magnets, based on Magcam's established 3-axis magnetic field camera technology. This method involves measuring the 3-axis magnetic field distribution in a 2D plane at close distance above the magnet and extrapolating this field distribution into a 3D volume using a patented algorithm that yields virtually noise-free data at larger distances. This powerful method allows to evaluate the magnetic field distribution and the magnet's intrinsic angular error, with a resolution better than the measurement resolution of the originally measured plane.
\end{abstract}

Keywords: sensor magnet, magnet inspection, magnetic field camera, magnetic field mapping, magnet

\section{Background, Motivation and Objective}

A method is presented for magnetically characterizing 2-pole rotary encoder magnets, which are widely used in angular encoders in e.g. electric motors and rotary positioning systems. The method uses a proprietary 'Distance Filter' algorithm, which allows to extrapolate a magnetic field distribution recorded at one certain (close) distance above a magnet or magnet assembly to another (larger) distance in a very fast way and with a strong suppression of measurement noise. This allows very fast and at the same time highly accurate measurements, making the method suitable for production quality control.

\section{Description of the Extrapolation Method}

The 'Distance Filter' extrapolation algorithm is used in combination with Magcam's magnetic field camera systems. When the extrapolation is in the direction away from the magnet, there is a strong suppression of noise, resulting in $\mu$ Tesla magnetic field resolutions. This makes the Distance Filter algorithm very powerful for determining magnetic field distributions far away from a magnet, since a direct measurement at the farther distance would suffer from a poorer signal-to-noise ratio.

For accurate results with the Distance Filter, the recorded magnetic field distribution must contain the full magnetic field of the measured magnet or magnet assembly, meaning that at all edges of the measured magnetic field image the magnetic field should be monotonically decreasing towards zero when going outwards towards the image edges. In practice, this means that a sufficiently large area should be measured, including extra space around the magnet.

\section{Results}

Consider a cylindrical axially magnetized 2-pole rotary encoder magnet, with the cylindrical symmetry axis pointing in the $Z$ direction. The same principle applies for diametrically magnetized cylinder magnets. The original measurement is in the $X Y$ plane at a certain height $Z 0$ above the magnet surface. The magnetic field distribution at a different height $\mathrm{Z1}$ is obtained by the Distance Filter method by supplying one single input parameter Delta, namely the distance between the original measurement plane and the desired plane: Delta $=\mathbf{Z 1}-\mathbf{Z 0}$. When Delta $=0$ the original data is retained.

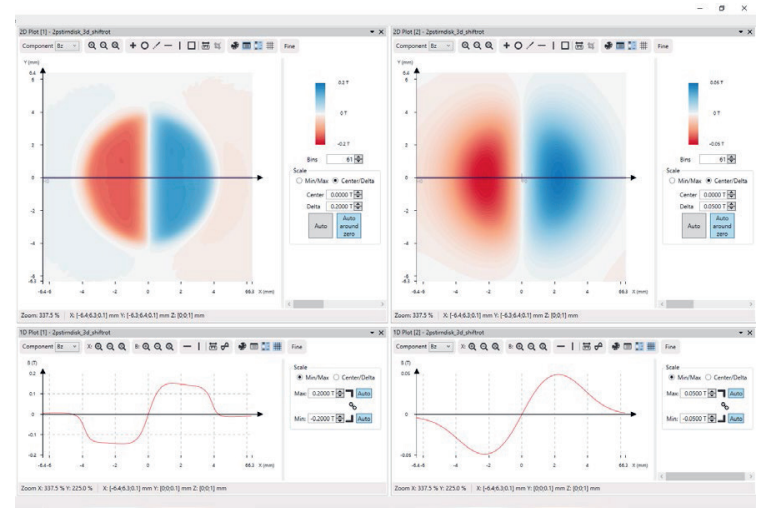

Fig. 1. Measured Bz magnetic field distribution and cross section along the $X$ direction at $Z 0=0.3 \mathrm{~mm}$ (left) and Distance Filter result at $Z 1=2 \mathrm{~mm}$ (right).

Fig. 1 shows the measured $\mathrm{Bz}$ magnetic field distribution and cross section along the $\mathrm{X}$ direc- 
tion at $Z 0=0.3 \mathrm{~mm}$ (left) and Distance Filter result at $Z 1=2 \mathrm{~mm}$ (right). Hereby the value for Delta is: Delta $=2 \mathrm{~mm}-0.3 \mathrm{~mm}=1.7 \mathrm{~mm}$.

\section{Analyzing the in-plane magnetic field and azimuthal angle error in 2-pole rotary en- coder magnets}

In typical end applications, a Bx,By sensor is positioned in the center of the rotary encoder magnet at a certain distance from the magnet surface, which is typically several $\mathrm{mm}$. At such distance the Bxy magnetic field is typically of the order of $50 \mathrm{mT}$. The Bx,By sensor then measures $B x$ and $B y$ and from those calculates the in-plane angle of the magnetic field using atan2(By,Bx). Inhomogeneities of the magnetic field distribution can cause errors on this measured angle value. During quality control on such magnets, this angle error needs to be determined with high accuracy, typically in the order of $0.1^{\circ}$ or better. By using a magnetic field camera in combination with the Distance Filter this can be achieved in a superior way as is shown below.

A magnetic field distribution measurement directly at the working distance of the Bx,By sensor in the above application would result in a poorer signal-to-noise ratio than a measurement very close to the magnet (typically 0.3$0.5 \mathrm{~mm}$ ). The Distance Filter makes it possible to 'preserve' the signal-to-noise ratio at close distance to remote distances, resulting in virtually noise-free magnetic field distributions.

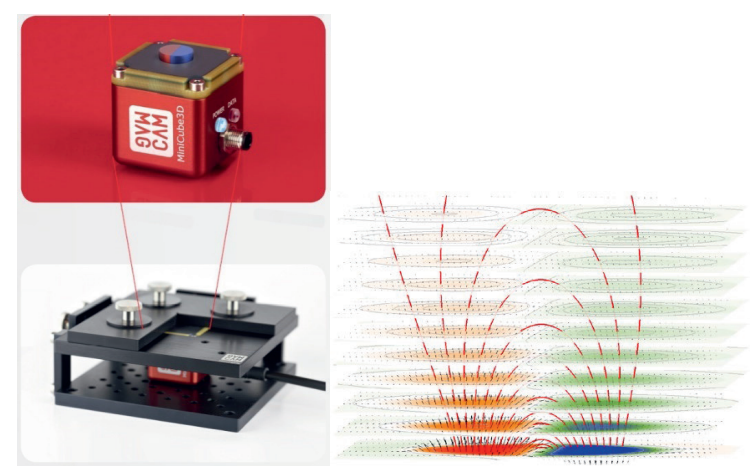

Fig. 2. 2-pole axially magnetized cylinder magnet measured with MiniCube3D magnetic field camera.

The 3D magnetic field distribution is measured at a close distance above the magnet surface using a Magcam MiniCube3D magnetic field camera (see Fig. 2).

As mentioned higher, the relevant magnetic field components for this application are the inplane (Bxy) magnetic field and the in-plane direction (azimuthal angle) of the field in a region in the center of the magnet. These components can be analyzed as explained below.

\section{Bxy field and azimuthal angle}

The Bxy component of the magnetic field distribution is readily obtained from the Bx and By components (Fig. 3). A circle is interpolated centered on the magnet center and with a certain radius, taken to be $0.25 \mathrm{~mm}$ in this example (see Figure 8), typically determined by the tolerance region where the Bx,By sensor will be located in the end application. In the obtained line plot, the maximum and minimum values can be automatically detected, which can be directly used in a pass/fail test to check if they are within the tolerance window.

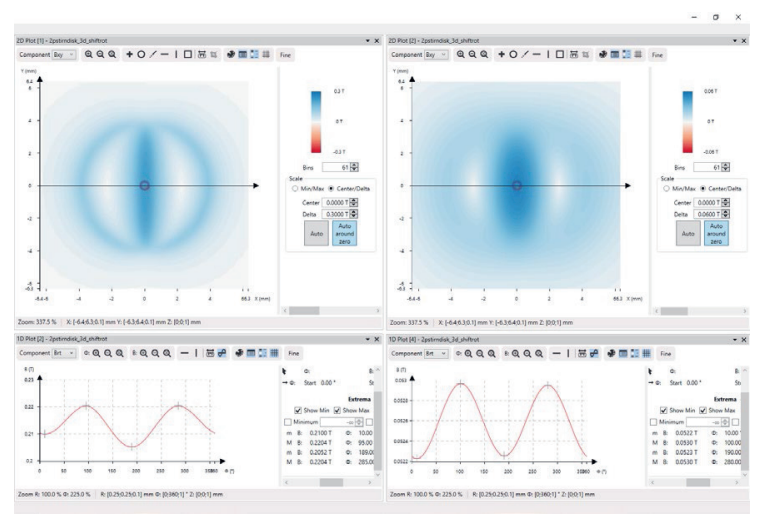

Fig. 3. Bxy (in-plane) magnetic field distribution with a circle section in the middle of the magnet for $Z$ $=0.3 \mathrm{~mm}$ (left) and $Z=2 \mathrm{~mm}$ (right). In the $1 \mathrm{D}$ Plots the minimum and maximum values are automatically detected.

The second important quantity of the magnetic field distribution is the in-plane direction (azimuthal angle) distribution of the magnetic field. This quantity is also readily calculated from the $B x$ and By components of the magnetic field by using atan2(By,Bx). By analyzing the extreme values of the azimuthal angle on the same circle section as before, the angle error is directly obtained, as shown in Fig. 4. By combining the results of Bxy and the azimuthal angle, a full quality control of rotary encoder magnets can be performed within seconds.

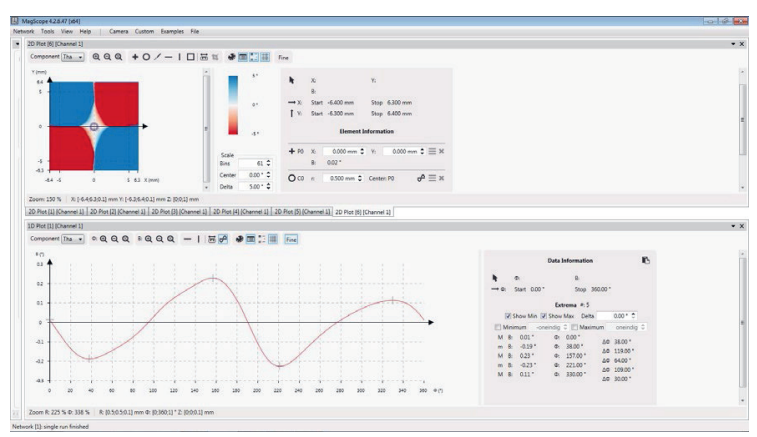

Fig. 4. Azimuthal (in-plane) angle distribution with a circle section in the middle of the magnet for $Z=$ $2 \mathrm{~mm}$. In the $1 \mathrm{D}$ Plot, the minimum and maximum values are automatically detected and give a direct measure of the angle error of the Bxy magnetic field distribution on the circle. 